\title{
PARABOLIC ITÔ EQUATIONS
}

\author{
BY \\ ROBERT MARCUS
}

ABSTRACT. A parabolic Itô equation is an equation of the form

$$
(\partial u / \partial t)(t, \omega)=L u(t, \omega)+f(u(t, \omega))+\alpha(t, \omega), \quad u(0)=u_{0}, u_{0}, u \in H .
$$

$H$ is a Hilbert space with scalar product $u \cdot v$ and norm $\mid . L$ is a linear time-independent negative-definite operator from $H$ to $H$. $f$ is a Lipschitz continuous operator from $H$ to $H$. $\alpha(t, \omega)$ is a white noise process in $H$.

Under suitable technical conditions the following results are obtained:

I. A unique nonanticipating solution of (1) exists with sup, $E\left\{|u|^{2}\right\}<\infty$.

II. $u(t, \omega)=R(t, \omega)+V(t, \omega)$ where $R(t, \omega)$ is a stationary process and

$$
\lim _{t \rightarrow \infty} E\left\{|V(t, \omega)|^{2}\right\}=0 .
$$

III. If $L$ is selfadjoint and $f$ is the gradient of a smooth functional then an explicit expression is found for the stationary density of $R(t, \omega)$ on $H$.

IV. For the equation $(\partial u / \partial t)(t, \omega)=L u(t, \omega)+f(u(t, \omega))+c \alpha(t, \omega)$ an asymptotic expansion in $\varepsilon$ is proven which holds uniformly in $t$.

I. Introduction. This paper is a first step toward a theory of random solutions of nonlinear partial differential equations. The basic heuristic idea is to find a class of equations whose solutions approach an "equilibrium state" as time goes to infinity. In many cases an explicit representation of the "equilibrium state" can be found even when the equation itself cannot be solved.

The most important application of the results in this paper would seem to be in the theory of random fluctuations of a system about an equilibrium.

A parabolic Itô equation is an equation of the form

$$
(\partial u / \partial t)(t, \omega)=L u(t, \omega)+f(u(t, \omega))+\alpha(t, \omega), \quad u(0)=u_{0}, u_{0}, u \in H .
$$

$H$ is a Hilbert space with scalar product $u \cdot v$ and norm $\mid \% . L$ is a linear timeindependent negative-definite operator from $H$ to $H . f$ is a Lipschitz continuous operator from $H$ to $H . \alpha(t, \omega)$ is a white noise process in $H$. Heuristically, this means that for every $v, u \in H, \alpha(t, \omega)$ satisfies

$$
E\{(\alpha(t, \omega) \cdot u)(\alpha(s, \omega) \cdot v)\}=(u \cdot v) \delta(t-s)
$$

where $E$ is the expectation in $\omega$.

Received by the editors April 16, 1973.

AMS (MOS) subject classifications (1970). Primary 60H15, 60H20.

Key words and phrases. Parabolic equations, Itô equations. 
II. An example. A simple example will first be discussed to illustrate the general theory. Let $u(x, t, \omega)$ satisfy

$$
\begin{gathered}
u_{t}=u_{x x}+\alpha(x, t, \omega), \\
u(x, 0)=0, \quad u(0, t)=0, \quad u_{x}(1, t)=0 .
\end{gathered}
$$

$\alpha(x, t, \omega)$ satisfies

$$
E\left\{\int_{0}^{1} f(x) \alpha(x, t, \omega) d x \int_{0}^{1} g(x) \alpha(x, s, \omega) d x\right\}=\delta(t-s) \int_{0}^{1} f(x) g(x) d x .
$$

Let $\phi_{i}(x)$ be a complete orthonormal set of vectors in $L^{2}(0,1)$ satisfying

$$
\frac{\partial^{2} \phi_{i}}{\partial x^{2}}=-\lambda_{i} \phi_{i}, \quad \phi_{i}(0)=0, \quad \frac{\partial \phi_{i}}{\partial x}(1)=0 .
$$

$u(x, t, \omega)$ can be expanded in terms of the orthonormal set $u(x, t, \omega)=\Sigma_{i} u_{i}(t$, $\omega) \phi_{i}(x)$. Similarly $\alpha(x, t, \omega)$ can be expanded: $\alpha(x, t, \omega)=\sum_{i} \alpha_{i}(t, \omega) \phi_{i}(x)$ where the $\alpha_{i}(t, \omega)$ are independent ordinary white noise processes.

Using the expansions the partial differential equation becomes

$$
d u_{i} / d t=-\lambda_{i} u_{i}+\alpha_{i}(t, \omega), \quad u_{i}(0)=0 .
$$

The solution to the system of equations is

$$
u_{i}(t, \omega)=\int_{0}^{t} \exp \left(-\lambda_{i}(t-s)\right) \alpha_{i}(s, \omega) d s .
$$

The $u_{i}(t, \omega)$ are Gaussian processes which satisfy

$$
\begin{aligned}
E\left\{u_{i}(t, \omega)\right\} & =0, \\
E\left\{u_{i}(t, \omega) u_{i}(s, \omega)\right\} & =\frac{\exp \left(-\lambda_{i}|t-s|\right)-\exp \left(-\lambda_{i}(t+s)\right)}{2 \lambda_{i}} .
\end{aligned}
$$

The above statements imply that $u_{i}(t, \omega)$ can be written in the following way:

$$
u_{i}(t, \omega)=R_{i}(t, \omega)+V_{i}(t, \omega) .
$$

$R_{i}(t, \omega)$ and $V_{i}(t, \omega)$ are Gaussian processes satisfying

$$
\begin{aligned}
E\left\{R_{i}(t, \omega)\right\} & =0, \quad E\left\{V_{i}(t, \omega)\right\}=0 \\
E\left\{R_{i}(t, \omega) R_{i}(s, \omega)\right\} & =\exp \left(-\lambda_{i}|t-s|\right) / 2 \lambda_{i}, \\
E\left\{R_{i}(t, \omega) V_{i}(s, \omega)\right\} & =-\exp \left(-\lambda_{i}(t+s)\right) / 2 \lambda_{i}, \\
E\left\{V_{i}(t, \omega) V_{i}(s, \omega)\right\} & =\exp \left(-\lambda_{i}(t+s)\right) / 2 \lambda_{i} .
\end{aligned}
$$

Note that $R_{i}(t, \omega)$ is a stationary process while $\lim _{t \rightarrow \infty} E\left\{V_{i}^{2}(t, \omega)\right\}=0$. Hence $\lim _{t \rightarrow \infty} E\left\{u_{i}^{2}(t, \omega)\right\}=E\left\{R_{i}^{2}(t, \omega)\right\}=1 / 2 \lambda_{i}$.

Recall $u(x, t, \omega)=\sum_{i=1}^{\infty} u_{i}(t, \omega) \phi_{i}(x)$ which implies that 


$$
\lim _{i \rightarrow \infty} E\{u(x, t, \omega) u(y, t, \omega)\}=\sum_{i=1}^{\infty} \frac{\phi_{i}(x) \phi_{i}(y)}{2 \lambda_{i}} .
$$

From the boundary conditions on $\phi_{i}(x)$,

$$
\sum_{i=1}^{\infty} \frac{\phi_{i}(x) \phi_{i}(y)}{2 \lambda_{i}}=\frac{1}{2} \min (x, y) .
$$

Combining all the above results it follows that as $t \rightarrow \infty, u(x, t, \omega)$ approaches a stationary process with a stationary measure on $L^{2}(0,1)$ equivalent to a Wiener measure.

The preceding discussion can be easily generalized to other selfadjoint elliptic operators on $L^{2}(D)$ where $D \subset R^{N}$. In particular for higher dimensions and suitable powers of the Laplacian, the stationary measure will correspond to a Wiener-Lévy process with multidimensional time.

III. Main results. The first step is to convert $(\partial u / \partial t)(t, \omega)=L u(t, \omega)+f(u(t, \omega))$ $+\alpha(t, \omega)$ into an integral equation with the kernel $P(t)$ which is the fundamental solution of $d P / d t=L P$. One obtains

$$
u(t, \omega)=\int_{0}^{t} P(t-s) f(u(s, \omega)) d s+\int_{0}^{t} P(t-s) \alpha(s, \omega) d s+P(t) u_{0} .
$$

Under suitable technical conditions the following results are obtained:

I. A unique nonanticipating solution of (1) exists with $\sup _{t} E\left\{|u|^{2}\right\}<\infty$.

II. $u(t, \omega)=R(t, \omega)+V(t, \omega)$ where $R(t, \omega)$ is a stationary process and $\lim _{t \rightarrow \infty} E\left\{|V(t, \omega)|^{2}\right\}=0$.

III. If $L$ is selfadjoint and $f$ is the gradient of a smooth functional then an explicit expression is found for the stationary density of $R(t, \omega)$ on $H$.

IV. For the equation $(\partial u / \partial t)(t, \omega)=L u(t, \omega)+f(u(t, \omega))+\varepsilon \alpha(t, \omega)$ an asymptotic expansion in $\varepsilon$ is proven which holds uniformly in $t$.

The following technical conditions are needed for the results of this paper:

(i) $f(u)$ is an operator from $H$ to $H$ satisfying $|f(u)-f(v)| \leq a|u-v|$.

(ii) For $t>0, P(t)$ is a Hilbert-Schmidt operator satisfying $|P(t)| \leq e^{-\lambda t}, a$ $<\lambda$, in which $\mid \cdot$ is the operator norm on $H$. This condition implies that $\int_{0}^{\infty}|P(t)| d t<1 / a$.

(iii) $\int_{0}^{\infty}$ Trace $\left(P^{*}(t) P(t)\right) d t=\sup _{t} E\left\{\left|\int_{0}^{t} P(t-s) \alpha(s, \omega) d s\right|^{2}\right\}<\infty$, where $P^{*}$ is the adjoint of $P$.

Note. If $L=A+i B$ where $A$ and $B$ are Hermitian operators satisfying

(1) $A+\left(B B^{*}\right)^{1 / 2}<-\lambda$,

(2) $\left(A+\left(B B^{*}\right)^{1 / 2}\right)^{-1}$ is of trace class,

then it is straightforward to show that $P(t)$ satisfies conditions (ii) and (iii) above.

Theorem 1. Under the technical conditions stated above, equation (1) has a unique nonanticipating solution $u(t, \omega) \in H$. Furthermore, $E\left\{|u(t, \omega)|^{2}\right\}$ is uniformly bounded in $t$. 
Note. Baklan [4] has announced a result similar to Theorem 1 except for the global bound on $E\left\{|u(t, \omega)|^{2}\right\}$.

Proof of Theorem 1. Using Picard iteration,

$$
u_{N+1}(t, \omega)=\int_{0}^{t} P(t-s) f\left(u_{N}(s, \omega)\right) d s+\int_{0}^{t} P(t-s) \alpha(s, \omega) d s+P(t) u_{0} .
$$

First it will be shown that $\lim _{N \rightarrow \infty} u_{N}(t, \omega)$ exists in the mean square sense uniformly in $t$.

Since

$$
u_{N+1}(t, \omega)-u_{N}(t, \omega)=\int_{0}^{t} P(t-s)\left(f\left(u_{N}\right)-f\left(u_{N-1}\right)\right) d s,
$$

then

$$
\sup _{t} E\left\{\left|u_{N+1}(t, \omega)-u_{N}(t, \omega)\right|^{2}\right\}=\sup _{t} E\left\{\left|\int_{0}^{t} d s P(t-s)\left(f\left(u_{N}\right)-f\left(u_{N-1}\right)\right)\right|^{2}\right\} .
$$

Now using conditions (i) and (ii),

$$
\begin{aligned}
\sup _{t} E & \left\{\left|\int_{0}^{t} d s P(t-s)\left(f\left(u_{N}\right)-f\left(u_{N-1}\right)\right)\right|^{2}\right\} \\
& \leq \sup _{t} E\left\{\left(\int_{0}^{t} d s|P(t-s)|\left|f\left(u_{N}\right)-f\left(u_{N-1}\right)\right|\right)^{2}\right\} \\
& \leq \sup _{t}\left(\int_{0}^{t} d s|P(t-s)|\right)^{2} \sup _{t} E\left\{\left|f\left(u_{N}\right)-f\left(u_{N-1}\right)\right|^{2}\right\} .
\end{aligned}
$$

(The last step comes from expanding the integral squared as a double integral and using obvious inequalities and manipulations.)

$$
\begin{aligned}
& \leq\left(\int_{0}^{\infty} d s|P(s)|\right)^{2} \sup _{t} a^{2} E\left\{\left|u_{N}-u_{N-1}\right|^{2}\right\} \\
& \leq \frac{a^{2}}{\lambda^{2}} \sup _{t} E\left\{\left|u_{N}-u_{N-1}\right|^{2}\right\}
\end{aligned}
$$

where $a^{2} / \lambda^{2}<1$.

This demonstrates that

$$
\sup _{t} E\left\{\left|u_{N+1}-u_{N}\right|^{2}\right\} \leq \frac{a^{2}}{\lambda^{2}} \sup _{t} E\left\{\left|u_{N}-u_{N-1}\right|^{2}\right\}
$$

Recall $a<\lambda$.

To complete the proof that $\lim _{N \rightarrow \infty} u_{N}$ converges, one must show that $\sup _{t} E\left\{\left|u_{1}-u_{0}\right|^{2}\right\}<\infty$.

Since $\left|u_{0}\right|<\infty$ the proof reduces to showing sup $E\left\{\left|u_{1}\right|^{2}\right\}<\infty$. Now

$$
u_{1}(t)=\int_{0}^{t} P(t-s) f\left(u_{0}\right) d s+\int_{0}^{t} P(t-s) \alpha(s, \omega) d s+P(t) u_{0},
$$


and therefore

$$
\begin{aligned}
\sup _{t} E\left\{\left|u_{1}\right|^{2}\right\} \leq & 3\left(\int_{0}^{\infty}|P(s)|^{2} d s\right)\left|f\left(u_{0}\right)\right|^{2} \\
& +3 \int_{0}^{\infty} \operatorname{Trace}\left(P^{*}(s) P(s)\right) d s+3\left|u_{0}\right|^{2}<\infty .
\end{aligned}
$$

The last statement follows from condition (iii).

The fact that $\sup _{t} E\left\{|u|^{2}\right\}<\infty$ follows from the Picard iteration and the convergence of the resulting geometric series. In fact the method used to prove Theorem 1 was chosen so that the global bound would be obtained simultaneously with the proof of existence and uniqueness of $u$. (Uniqueness follows from the standard argument for Picard iteration.)

Theorem 2. The solution of equation (1) can be split as follows: $u(t, \omega)=R(t, \omega)$ $+V(t, \omega)$ where $R(t, \omega)$ is a stationary process and $V(t, \omega)$ satisfies

$$
\lim _{t \rightarrow \infty} E\left\{\mid V(t, \omega)^{2}\right\}=0 .
$$

Note. The following argument shows that $R(t, \omega)$ is independent of $u_{0}$, i.e., the stationary distribution of $u$ is unique. The conditions of Theorem 1 imply that $L u+f(u)$ is a monotone operator, i.e., $(L u+f(u)-L v-f(v)) \cdot(u-v)<(a$ $-\lambda)|u-v|^{2}$. Let $u$ and $v$ satisfy equation (1) with initial conditions $u_{0}$ and $v_{0}$ respectively. Then it is easily shown that

$$
(\partial / \partial t)\left(\frac{1}{2}|u-v|^{2}\right)=(L u-L v+f(u)-f(v)) \cdot(u-v)<(a-\lambda)|u-v|^{2} .
$$

This implies that $\lim _{t \rightarrow \infty}|u-v|=0$.

Proof of Theorem 2. There are two parts to the proof. First it is shown by induction that each $u_{N}(t, \omega)$ in the Picard iteration can be split in the above manner. Then splitting of $u(t, \omega)$ is accomplished by taking limits.

Since $u_{0}(t, \omega)=u_{0}$, let $R_{0}(t, \omega)=u_{0}$ and $V_{0}(t, \omega)=0$. Note that $R_{0}(t, \omega)$ is defined for $-\infty<t<\infty$. Assume that $u_{N}(t, \omega)=R_{N}(t, \omega)+V_{N}(t, \omega)$ where $R_{N}(t, \omega)$ is a stationary process defined for $-\infty<t<\infty$ and

$$
\lim _{t \rightarrow \infty} E\left\{\left|V_{N}(t, \omega)\right|^{2}\right\}=0 .
$$

Now

$$
\begin{aligned}
u_{N+1}(t, \omega)= & \int_{0}^{t} P(t-s) f\left(u_{N}(s, \omega)\right) d s+\int_{0}^{t} P(t-s) \alpha(s, \omega) d s+P(t) u_{0} \\
= & \int_{-\infty}^{t} P(t-s) f\left(R_{N}\right) d s+\int_{-\infty}^{t} P(t-s) \alpha(s, \omega) d s \\
& -\int_{-\infty}^{0} P(t-s) f\left(R_{N}\right) d s-\int_{-\infty}^{0} P(t-s) \alpha(s, \omega) d s \\
& +P(t) u_{0}+\int_{0}^{t} P(t-s)\left(f\left(u_{N}\right)-f\left(R_{N}\right)\right) d s .
\end{aligned}
$$


Let

$$
R_{N+1}(t, \omega)=\int_{-\infty}^{t} P(t-s) f\left(R_{N}\right) d s+\int_{-\infty}^{t} P(t-s) \alpha(s, \omega) d s
$$

and

$$
\begin{aligned}
V_{N+1}(t, \omega)= & -\int_{-\infty}^{0} P(t-s) f\left(R_{N}\right) d s-\int_{-\infty}^{0} P(t-s) \alpha(s, \omega) d s \\
& +P(t) u_{0}+\int_{0}^{t} P(t-s)\left(f\left(u_{N}\right)-f\left(R_{N}\right)\right) d s .
\end{aligned}
$$

Note $R_{N+1}(t, \omega)$ is a stationary process.

The problem is to verify $\lim _{t \rightarrow \infty} E\left\{\left|V_{N+1}(t, \omega)\right|^{2}\right\}=0$. The last term in the expression for $V_{N+1}(t, \omega)$ is $\int_{0}^{t} P(t-s)\left(f\left(u_{N}\right)-f\left(R_{N}\right)\right) d s$. It is easy to use the methods of Theorem 1 to prove that

$$
E\left\{\left|\int_{0}^{t} P(t-s)\left(f\left(u_{N}\right)-f\left(R_{N}\right)\right) d s\right|^{2}\right\} \leq \frac{a^{2}}{\lambda^{2}} \sup _{t} E\left\{\left|V_{N}\right|^{2}\right\} .
$$

Now if the three other terms which comprise $V_{N+1}(t, \omega)$ can be shown to approach 0 as $t \rightarrow \infty$ at a rate independent of $N$, then the decomposition of $u_{N}$ will be completed.

The first term is $\int_{-\infty}^{0} P(t-s) f\left(R_{N}\right) d s$. Using the methods of Theorem 1, it can be shown that

$$
E\left\{\left|\int_{-\infty}^{0} P(t-s) f\left(R_{N}(s)\right) d s\right|^{2}\right\} \leq\left(\int_{-\infty}^{0}|P(t-s)| d s\right)^{2} \sup _{-\infty<i<0} E\left\{\left|f\left(R_{N}\right)\right|^{2}\right\} .
$$

From Theorem 1 it follows that $\sup _{t} E\left\{\left|f\left(u_{N}\right)\right|^{2}\right\}$ is bounded uniformly in $N$. Since $\lim _{t \rightarrow \infty} E\left\{\left|f\left(u_{N}\right)\right|^{2}\right\}=E\left\{\left|f\left(R_{N}\right)\right|^{2}\right\}, \quad \sup _{-\infty<t<0} E\left\{\left|f\left(R_{N}\right)\right|^{2}\right\}<c<\infty$. Hence

$$
E\left\{\left|\int_{-\infty}^{0} P(t-s) f\left(R_{N}(s)\right) d s\right|^{2}\right\} \leq c\left(\int_{-\infty}^{0}|P(t-s)| d s\right)^{2} .
$$

The right-hand side vanishes uniformly in $N$ as $t \rightarrow \infty$.

For the second term

$$
E\left\{\left|\int_{-\infty}^{0} P(t-s) \alpha(s, \omega) d s\right|^{2}\right\}=\int_{t}^{\infty} \operatorname{Trace}\left(P^{*}(s) P(s)\right) d s .
$$

By condition (iii) preceding Theorem 1, the right-hand side approaches 0 as $t \rightarrow \infty$.

For the third term $E\left\{\left|P(t) u_{0}\right|^{2}\right\} \leq\left|u_{0}\right|^{2}|P(t)|^{2} \leq\left|u_{0}\right|^{2} e^{-2 \lambda}$. Again the righthand side vanishes as $t \rightarrow \infty$. This completes the proof that

uniformly in $N$.

$$
\lim _{t \rightarrow \infty} E\left\{\left|V_{N+1}(t, \omega)\right|^{2}\right\}=0
$$


It has been shown that $u_{N}(t, \omega)=R_{N}(t, \omega)+V_{N}(t, \omega)$. To complete the proof of Theorem 2 the decomposition must be extended to $\lim _{N \rightarrow \infty} u_{N}(t, \omega)=u(t, \omega)$.

The first step is to show that $\lim _{N \rightarrow \infty} R_{N}(t, \omega)=R(t, \omega)$. The proof is by contradiction; assume there exists an $\varepsilon$ such that, for all $n$,

$$
\sup _{M, N>n} E\left\{\left|R_{N}(t, \omega)-R_{M}(t, \omega)\right|^{2}\right\}>2 \varepsilon .
$$

Since the difference of two stationary processes is stationary, the expression on the left is independent of time. By choosing $t$ large enough, thus making $E\left\{\left|V_{N}(t, \omega)\right|^{2}\right\}$ and $E\left\{\left|V_{M}(t, \omega)\right|^{2}\right\}$ sufficiently small, it is easy to show $\sup _{t} \sup _{M, N>n} E\left\{\left|u_{N}-u_{M}\right|^{2}\right\}>\varepsilon$. But this contradicts the result that $\lim _{N \rightarrow \infty} u_{N}(t$, $\omega)$ exists, which was proven in Theorem 1. Since $R(t)=\lim _{N \rightarrow \infty} R_{N}(t)$ is the limit of stationary processes, it is a stationary process.

The final step in proving Theorem 2 is to show

$$
\lim _{t \rightarrow \infty} E\left\{|u(t, \omega)-R(t, \omega)|^{2}\right\}=0 .
$$

Using a standard inequality,

$$
\begin{aligned}
E\left\{|u(t, \omega)-R(t, \omega)|^{2}\right\} \leq & 3 E\left\{\left|u(t, \omega)-u_{N}(t, \omega)\right|^{2}\right\} \\
& +3 E\left\{\left|u_{N}(t, \omega)-R_{N}(t, \omega)\right|^{2}\right\}+3 E\left\{\left|R_{N}(t, \omega)-R(t, \omega)\right|^{2}\right\} .
\end{aligned}
$$

Take $N$ large enough so that the first and third terms are less than $\varepsilon / 3$ uniformly in $t$. Then choose $t$ large enough so that $E\left\{\left|u_{N}-R_{N}\right|^{2}\right\}<\varepsilon / 3$. Combining the three terms one has shown $E\left\{|u(t, \omega)-R(t, \omega)|^{2}\right\}<\varepsilon$ for sufficiently large $t$. This completes the proof of Theorem 2 that $u(t, \omega)=R(t, \omega)+V(t, \omega)$ where $R(t, \omega)$ is a stationary process and $\lim _{t \rightarrow \infty} E\left\{|V(t, \omega)|^{2}\right\}=0$.

Note that for $f(u)=0$, the stationary measure for $R(t, \omega)$ is a Gaussian measure with mean 0 and covariance $\int_{0}^{\infty} P^{*}(s) P(s) d s$. Writing the covariance in the form $\int_{0}^{\infty} e^{s L} e^{s L^{*}} d s$ gives an explicit representation $-\left(L+L^{*}\right)^{-1}$ when $L$ is a normal operator. If $L$ and $L^{*}$ do not commute, no simple general expression can be found for the covariance.

Even if $f(u) \neq 0$, it may still be possible to find the stationary distribution explicitly. If $L$ is selfadjoint, let $\mathfrak{p}_{0}$ be the Gaussian distribution with mean 0 and covariance operator $-\frac{1}{2} L^{-1}$. ( $L^{-1}$ exists because $L$ is a selfadjoint negative-definite operator.) Then if $F(u)$ is an indefinite Fréchet integral of $f(u)$, it will be proved that the stationary distribution $\mathfrak{p}$ of $u(t, \omega)$ is described by the following formula:

$$
\begin{aligned}
\lim _{t \rightarrow \infty} E\{G(u(t, \omega))\} & =\int_{H} G(u) \delta \mathfrak{p}(u) \\
& =\int_{H} G\left(u_{0}\right) e^{2 F\left(u_{0}\right)} \delta \mathfrak{p}_{0}\left(u_{0}\right) / \int_{H} e^{2 F\left(u_{0}\right)} \delta \mathfrak{p}_{0}\left(u_{0}\right) .
\end{aligned}
$$

$\int_{H} G(u) \delta \mathfrak{p}(u)$ is the integral of $G(u)$ over $H$ with respect to the distribution $\mathfrak{p} . G$ is any bounded measurable functional on $H$. In other words, $\mathfrak{p}$ has RadonNikodym derivative $e^{2 F(u)} / \int_{H} e^{2 F(u)} \delta \mathfrak{p}_{0}(u)$ with respect to $\mathfrak{p}_{0}$. 
Theorem 3. Let $u(t, \omega)$ satisfy all the conditions of Theorem 1. Then the stationary distribution of $u(t, \omega)$ is the distribution $\mathfrak{p}$ described above.

Proof of Theorem 3. The proof has two parts. First a sequence $u_{N}$ is given, converging uniformly in $t$ to $u$. The stationary distributions of $u_{N}$ can be found explicitly. Finally the stationary distributions of $u_{N}$ will be shown to converge to $\mathfrak{p}$.

Recall $L$ is a selfadjoint linear operator with an inverse of trace class. Hence $L$ has a complete set of orthonormal eigenvectors $\phi_{i}$ such that $L \phi_{i}=\lambda_{i} \phi_{i}$. Let $J_{N}$ be the projection of $H$ onto the finite-dimensional vector space spanned by $\left\{\phi_{i}\right\}$ for $i=1,2,3, \ldots, N$. Note the equation $d P / d t=L P$ implies $\left|P(t)-P(t) J_{N}\right|$ $\leq c e^{-\lambda_{N} t}$ for some constant $c$.

Let $u_{N}(t, \omega)$ be the solution of

$$
u_{N}(t)=\int_{0}^{t} P(t-s) J_{N} f\left(J_{N} u_{N}\right) d s+\int_{0}^{t} P(t-s) \alpha(s, \omega) d s+P(t) u_{0} .
$$

Recall $u(t)=\int_{0}^{t} P(t-s) f(u) d s+\int_{0}^{t} P(t-s) \alpha(s, \omega) d s+P(t) u_{0}$.

Now it will be shown that $u_{N}$ converges to $u$ uniformly in $t$.

$$
\begin{aligned}
\lim _{N \rightarrow \infty} \sup _{t} E\left\{\left|u-u_{N}\right|\right\} & =\lim _{N \rightarrow \infty} \sup _{t} E\left\{\left|\int_{0}^{t} P(t-s)\left(f(u)-J_{N} f\left(J_{N} u_{N}\right)\right) d s\right|\right\} \\
\leq & \lim _{N \rightarrow \infty} \sup _{t} E\left\{\left|\int_{0}^{t} P(t-s)\left(f(u)-f\left(u_{N}\right)\right) d s\right|\right\} \\
& +\lim _{N \rightarrow \infty} \sup _{t} E\left\{\left|\int_{0}^{t} P(t-s)\left(f\left(u_{N}\right)-f\left(J_{N} u_{N}\right)\right) d s\right|\right\} \\
& +\lim _{N \rightarrow \infty} \sup _{t} E\left\{\left|\int_{0}^{t} P(t-s)\left(f\left(J_{N} u_{N}\right)-J_{N} f\left(J_{N} u_{N}\right)\right)\right| d s\right\} \\
\leq & \lim _{N \rightarrow \infty} \sup _{t} \int_{0}^{t}|P(t-s)| E\left\{\left|f(u)-f\left(u_{N}\right)\right|\right\} d s \\
& +\lim _{N \rightarrow \infty} \sup _{t} \int_{0}^{t}|P(t-s)| E\left\{\left|f\left(u_{N}\right)-f\left(J_{N} u_{N}\right)\right|\right\} d s \\
& +\lim _{N \rightarrow \infty} \sup _{t} \int_{0}^{t}\left|P(t-s)-P(t-s) J_{N}\right| E\left\{\left|f\left(J_{N} u_{N}\right)\right|\right\} d s \\
\leq & \lim _{N \rightarrow \infty} \sup _{t} \frac{a}{\lambda} E\left\{\left|u-u_{N}\right|\right\} \\
& +\lim _{N \rightarrow \infty} \sup _{t} \frac{a}{\lambda} E\left\{\left|u_{N}-J_{N} u_{N}\right|\right\}+\lim _{N \rightarrow \infty} \sup _{t} \frac{a}{\lambda_{N}} E\left\{\left|f\left(J_{N} u_{N}\right)\right|\right\} .
\end{aligned}
$$

Simple algebra yields

$$
\begin{aligned}
\left(1-\frac{a}{\lambda}\right) & \lim _{N \rightarrow \infty} \sup _{t} E\left\{\left|u-u_{N}\right|\right\} \\
& \leq \frac{a}{\lambda} \lim _{N \rightarrow \infty} \sup _{t} E\left\{\left|u_{N}-J_{N} u_{N}\right|\right\}+\lim _{N \rightarrow \infty} \frac{a}{\lambda_{N}} \sup E\left\{\left|f\left(J_{N} u_{N}\right)\right|\right\} .
\end{aligned}
$$


For the first term on the right-hand side of the inequality, using the equation

$$
u_{N}(t)-J_{N} u_{N}(t)=\int_{0}^{t} P(t-s)\left(\alpha(s)-J_{N} \alpha(s)\right) d s+P(t) u_{0}-P(t) J_{N} u_{0}
$$

it is easy to show that the limit is equal to 0 .

For the second term, using the equation

$$
J_{N} u_{N}(t)=\int_{0}^{t} P(t-s) J_{N} f\left(J_{N} u_{N}\right) d s+\int_{0}^{t} P(t-s) J_{N} \alpha(s) d s+P(t) J_{N} u_{0}
$$

and an argument duplicating the proof of Theorem 1, it is straightforward to show that $\sup _{t} E\left\{\left|u_{N}\right|\right\}$ is bounded uniformly in $N$. Then

$$
\lim _{N \rightarrow \infty} \frac{a}{\lambda_{N}} \sup _{t} E\left\{\left|f\left(J_{N} u_{N}\right)\right|\right\} \leq \lim _{N \rightarrow \infty} \frac{a}{\lambda_{N}} \sup _{t} E\left\{a\left|u_{N}\right|+\left|J_{N} f(0)\right|\right\}=0 .
$$

This completes the proof that $\lim _{N \rightarrow \infty} \sup _{t} E\left\{\left|u-u_{N}\right|\right\}=0$.

The stationary distribution of $u_{N}$ can be found explicitly in two steps. $u_{N}=J_{N} u_{N}+\left(u_{N}-J_{N} u_{N}\right)$. On $J_{N} H$,

$$
J_{N} u_{N}(t)=\int_{0}^{t} P(t-s) J_{N} f\left(J_{N} u_{N}\right) d s+\int_{0}^{t} P(t-s) J_{N} \alpha(s) d s+P(t) J_{N} u_{0} .
$$

Since $J_{N} H$ is a finite-dimensional vector space, the unique stationary distribution of $J_{N} u_{N}$ can be found explicitly. [See Appendix.] The stationary distribution of $J_{N} u_{N}$ has a Radon-Nikodym derivative with respect to $\mathfrak{p}_{0}$ on $J_{N} H$ equal to $\exp \left(2 F\left(J_{N} u\right)\right) / \int_{H} \exp \left(2 F\left(J_{N} u\right)\right) \delta p_{0}(u)$. Note the Fréchet derivative of $F\left(J_{N} u\right)$ is $J_{N} f\left(J_{N} u\right)$.

The stationary distribution of $u_{N}-J_{N} u_{N}$ is easily determined using the equation

$$
u_{N}-J_{N} u_{N}=\int_{0}^{t} P(t-s)\left(\alpha(s)-J_{N} \alpha(s)\right) d s+P(t) u_{0}-P(t) J_{N} u_{0} .
$$

The stationary distribution of $u_{N}-J_{N} u_{N}$ is a Gaussian measure with covariance operator $\left(2 L-2 L J_{N}\right)^{-1}$. Combining these two results, the stationary distribution of $u_{N}$ has the Radon-Nikodym derivative $\exp \left(2 F\left(J_{N} u\right)\right) / \int_{H} \exp \left(2 F\left(J_{N} u\right)\right) \delta p_{0}$ with respect to $\mathfrak{p}_{0}$.

Since $\lim _{N \rightarrow \infty} \sup _{t} E\left\{\left|u_{N}-u\right|\right\}=0$ the stationary distribution of $u$ is the limit of the stationary distributions of $u_{N}$. To complete the proof it must be shown that the stationary distributions of $u_{N}$ converge to the measure $\mathfrak{p}$ with RadonNikodym derivative $\exp (2 F(u)) / \int_{H} \exp (2 F(u)) \delta p_{0}(u)$ with respect to $\mathfrak{p}_{0}$.

It is sufficient to show that

$$
\lim _{N \rightarrow \infty} \int_{H}\left|\exp (2 F(u))-\exp \left(2 F\left(J_{N} u\right)\right)\right| \delta \mathfrak{p}_{0}(u)=0
$$

since this implies weak convergence. 


$$
\begin{aligned}
\lim _{N \rightarrow \infty} & \int_{H}\left|\exp (2 F(u))-\exp \left(2 F\left(J_{N} u\right)\right)\right| \delta \mathfrak{p}_{0}(u) \\
& =\lim _{N \rightarrow \infty} \int_{H}\left|\exp \left(2 F(u)-2 F\left(J_{N} u\right)\right)-1\right|\left|\exp \left(2 F\left(J_{N} u\right)\right)\right| \delta \mathfrak{p}_{0}(u) \\
& \leq \lim _{N \rightarrow \infty} \int_{H}\left(\exp \left(a\left|u-J_{N} u\right|^{2}\right)-1\right)\left(\exp \left(a\left|J_{N} u\right|^{2}+2 F(0)\right)\right) \delta \mathfrak{p}_{0}(u) \\
& =\lim _{N \rightarrow \infty} \int_{H}\left(\exp \left(a\left|u-J_{N} u\right|^{2}\right)-1\right) \delta \mathfrak{p}_{0}(u) \int_{H} \exp \left(a\left|J_{N} u\right|^{2}+2 F(0)\right) \delta \mathfrak{p}_{0}(u) \\
& \leq \lim _{N \rightarrow \infty}\left|\prod_{i=N+1}^{\infty}\left(\lambda_{i} /\left(\lambda_{i}-a\right)\right)^{1 / 2}-1\right| \exp (2 F(0)) \prod_{i=1}^{N}\left(\lambda_{i} /\left(\lambda_{i}-a\right)\right)^{1 / 2} \\
& =0 .
\end{aligned}
$$

(The last step is a consequence of the convergence of $\left.\prod_{i=1}^{\infty}\left(\lambda_{i} / \lambda_{i}-a\right)\right)^{1 / 2}$ which follows easily from the fact that $L^{-1}$ is of trace class.) This completes the proof of Theorem 3.

Theorem 4. Let $u(t, \omega)$ satisfy $u(t, \omega)=\int_{0}^{t} P(t-s) f(u(s, \omega)) d s+P(t) \hat{u}_{0}$ $+\varepsilon \int_{0}^{t} P(t-s) \alpha(s, \omega) d s$ and all conditions of Theorem 1 .

Let $f(u)$ have the following expansion for any $u_{0}, u_{1}, \ldots, u_{N} \in H$. Define $h=\varepsilon u_{1}+\varepsilon^{2} u_{2}+\cdots+\varepsilon^{N} u_{N}$. Then

$$
\begin{aligned}
f\left(u_{0}+h\right)= & f\left(u_{0}\right)+f^{\prime}\left(u_{0}\right) h_{*}+\frac{f^{\prime \prime}\left(u_{0}\right)}{2}\left(h^{2}\right)_{*} \\
& +\cdots+\frac{f^{N}\left(u_{0}\right)}{N !}\left(h^{N}\right)_{*}+R_{1}\left(u_{0}, u_{1}, \ldots, u_{N}, \varepsilon\right),
\end{aligned}
$$

where the subscript $t_{*}$ means keep terms only up to order $\varepsilon^{N}$. Let $R_{1}$ satisfy

$$
R_{1}\left(u_{0}, u_{1}, \ldots, u_{N}, \varepsilon\right) \leq C\left(\left|u_{0}\right|,\left|u_{1}\right|, \ldots,\left|u_{N}\right|\right) \varepsilon^{N+1}
$$

where $C$ has at most polynomial growth in $\left|u_{0}\right|,\left|u_{1}\right|, \ldots,\left|u_{N}\right|$. (In general this is an expansion in functional derivatives and $f^{N}(u)$ will be a bounded $N$-linear operator on $H^{N}$.)

Then

$$
u(t, \omega)=u_{0}(t)+\varepsilon u_{1}(t, \omega)+\cdots+\varepsilon^{N} u_{N}(t, \omega)+R_{2}(t, \omega, \varepsilon)
$$

where $\lim _{\varepsilon \rightarrow 0} \sup _{t} E\left\{\left|R_{2}(t, \omega, \varepsilon)\right|\right\} / \varepsilon^{N}=0$.

The $u_{i}(t)$ can be found by using the expansion for $f(u)$ and matching terms in the equation for $u(t, \omega)$. For example,

$$
\begin{aligned}
u_{0}(t) & =\int_{0}^{t} P(t-s) f\left(u_{0}(s)\right) d s+P(t) \hat{u}_{0}, \\
u_{1}(t, \omega) & =\int_{0}^{t} P(t-s) f^{\prime}\left(u_{0}(s)\right) u_{1}(s, \omega) d s+\int_{0}^{t} P(t-s) \alpha(s, \omega) d s, \\
u_{2}(t, \omega) & =\int_{0}^{t} P(t-s) f^{\prime}\left(u_{0}\right) u_{2}(s, \omega) d s+\int_{0}^{t} P(t-s) \frac{f^{\prime \prime}\left(u_{0}\right)}{2} u_{1}^{2}(s, \omega) d s .
\end{aligned}
$$


Remark. Using the above equations it is possible to show $E\left\{\left|u_{i}(t, \omega)\right|^{M}\right\}<\infty$ where $i>1$ and $M$ is any integer. This result will be needed in the proof of Theorem 4.

The proof, which is straightforward but involved, is by showing inductively that $E\left\{\left|u_{i}(t, \omega)\right|^{M}\right\}$ is less than an expectation involving only functionals of polynomial growth in $u_{1}$. Since $u_{1}(t, \omega)$ is a Gaussian random element in $H$ as can be seen from its defining equation, the expectation is finite.

Proof of Theorem 4.

$$
\begin{aligned}
R_{2}(t, \omega, \varepsilon)= & u-u_{0}-\varepsilon u_{1}-\varepsilon^{2} u_{2}-\cdots-\varepsilon^{N} u_{N} \\
= & \int_{0}^{t} P(t-s)\left(f(u)-f\left(u_{0}\right)-\varepsilon f^{\prime}\left(u_{0}\right) u_{1}-\cdots-\text { term of order } \varepsilon^{N}\right) d s \\
= & \int_{0}^{t} P(t-s)\left(f(u)-f\left(u_{0}+\varepsilon u_{1}+\cdots+\varepsilon^{N} u_{N}\right)\right. \\
& \left.\quad+R_{1}\left(u_{0}, u_{1}, u_{2}, \ldots, u_{N}, \varepsilon\right)\right) d s \\
= & \int_{0}^{t} P(t-s)\left(f(u)-f\left(u_{0}+\varepsilon u_{1}+\cdots+\varepsilon^{N} u_{N}\right)\right) d s \\
& +\int_{0}^{t} P(t-s) R_{1}\left(u_{0}, u_{1}, u_{2}, \ldots, u_{N}, \varepsilon\right) d s .
\end{aligned}
$$

Using the triangle inequality,

$$
\begin{aligned}
& \sup _{t} E\left\{\left|R_{2}(t, \omega)\right|\right\} \\
& \leq \sup _{t} E\left\{\left|\int_{0}^{t} P(t-s)\left(f(u)-f\left(u_{0}+\varepsilon u_{1}+\cdots+\varepsilon^{N} u_{N}\right)\right) d s\right|\right\} \\
& \quad+\sup _{t} E\left\{\left|\int_{0}^{t} P(t-s) R_{1}(s) d s\right|\right\} .
\end{aligned}
$$

Using the conditions of Theorem 1 it is not difficult to show for the first term

$$
\begin{aligned}
\sup _{t} E & \left\{\left|\int_{0}^{t} P(t-s)\left(f(u)-f\left(u_{0}+\varepsilon u_{1}+\cdots+\varepsilon^{N} u_{N}\right)\right) d s\right|\right\} \\
& \leq \sup _{t} \int_{0}^{t}|P(t-s)| E\left\{\left|f(u)-f\left(u_{0}+\varepsilon u_{1}+\cdots+\varepsilon^{N} u_{N}\right)\right| d s\right\} \\
& \leq \frac{a}{\lambda} \sup _{t} E\left\{\left|u-u_{0}-\varepsilon u_{1}-\cdots-\varepsilon^{N} u_{N}\right|\right\}=\frac{a}{\lambda} \sup _{t} E\left\{\left|R_{2}(t, \omega)\right|\right\} .
\end{aligned}
$$

For the second term,

$$
\begin{aligned}
& \sup _{t} E\left\{\left|\int_{0}^{t} P(t-s) R_{1}(s) d s\right|\right\} \leq \sup _{t} E\left\{\int_{0}^{t}|P(t-s)|\left|R_{1}(s)\right| d s\right\} \\
& \leq \frac{1}{\lambda} \sup _{t} E\left\{\left|R_{1}\left(u_{0}(t), u_{1}(t), \ldots, u_{N}(t), \varepsilon\right)\right|\right\} \leq \frac{\varepsilon^{N+1}}{\lambda} \sup _{t} E\left\{C\left(\left|u_{0}\right|,\left|u_{1}\right|,\left|u_{2}\right|, \ldots,\left|u_{N}\right|\right)\right\} \\
& \leq \frac{c_{0} \varepsilon^{N+1}}{\lambda} \text { with a constant } c_{0}, \text { independent of }|\varepsilon|<1 .
\end{aligned}
$$


The fact that $\sup _{t} E\left\{C\left(\left|u_{0}\right|,\left|u_{1}\right|,\left|u_{2}\right|, \ldots,\left|u_{N}\right|\right)\right\}$ is finite follows from combining the polynomial growth condition on $C$ given in the statement of Theorem 4 and the result mentioned in the remark that $\sup _{t} E\left\{\left|u_{i}(t, \omega)\right|^{M}\right\}<\infty$.

Combining the two inequalities just derived, one obtains

$$
\sup _{t} E\left\{\left|R_{2}(t, \omega)\right|\right\} \leq \frac{c_{0}}{\lambda} \varepsilon^{N+1}+\frac{a}{\lambda} \sup _{t} E\left\{\left|R_{2}(t, \omega)\right|\right\} .
$$

Simple algebra yields

$$
\sup _{t} E\left\{\left|R_{2}(t, \omega)\right|\right\} \leq \frac{c_{0}}{\lambda} \varepsilon^{N+1} /\left(1-\frac{a}{\lambda}\right) .
$$

Since $a / \lambda<1$

$$
\lim _{e \rightarrow 0} \frac{1}{\varepsilon^{N}} E\left\{\left|R_{2}(t, \omega)\right|\right\}=0
$$

completing the proof of Theorem 4.

Schilder [2] has obtained by entirely different methods results of the form:

$$
\lim _{\varepsilon \rightarrow 0} \int_{H} G(\varepsilon u) \exp \left(\varepsilon^{-2} F(\varepsilon u)\right) \delta \mathfrak{w}(u) / \int_{H} \exp \left(\varepsilon^{-2} F(\varepsilon u)\right) \delta \mathfrak{w}(u)=G\left(u^{*}\right) .
$$

Here $\mathfrak{w}(u)$ is the Wiener measure on $H, u^{*}$ is a minimum point for a certain functional, $\operatorname{grad} F$ satisfies a Lipschitz condition, and $G$ satisfies a smoothness condition at $u^{*}$. Note that the ratio is actually the expectation of $G$ with respect to a measure which is absolutely continuous with respect to Wiener measure.

Schilder's result can be duplicated using the methods of this paper. Let

$$
u_{t}^{e}(x, t, \omega)=u_{x x}^{e}(x, t, \omega)+\frac{1}{2} \operatorname{grad} F\left(u^{e}\right)+\varepsilon \alpha(x, t, \omega)
$$

with appropriate side conditions.

From Theorem 3 it can be shown, for smooth $G$,

$$
\lim _{t \rightarrow \infty} G\left(u^{e}(x, t)\right)=\int_{H} G(\varepsilon u) \exp \left(\varepsilon^{-2} F(\varepsilon u)\right) \delta \mathfrak{w}(u) / \int_{H} \exp \left(\varepsilon^{-2} F(\varepsilon u)\right) \delta \mathfrak{w}(u) .
$$

From Theorem 4, $\lim _{e \rightarrow 0} u^{e}(x, t, \omega)=u_{0}(x, t)$ uniformly in $t$ where $u_{0}(x, t)$ satisfies $\partial u_{0} / \partial t=u_{0 x x}+\frac{1}{2} \operatorname{grad} F\left(u_{0}\right)$. It is easy to show $\lim _{t \rightarrow \infty} u_{0}(x, t)=u^{*}(x)$ where $u^{*}(x)$ satisfies $u_{x x}^{*}+\frac{1}{2} \operatorname{grad} F\left(u^{*}\right)=0$. This $u^{*}(x)$ corresponds to the $u^{*}$ in Schilder's result. Finally

$$
\begin{array}{rl}
\lim _{\varepsilon \rightarrow 0} \int_{H} & G(\varepsilon u) \exp \left(\varepsilon^{-2} F(\varepsilon u)\right) \delta \mathrm{m}(u) / \int_{H} \exp \left(\varepsilon^{-2} F(\varepsilon u)\right) \delta \mathrm{rm}(u) \\
= & \lim _{\varepsilon \rightarrow 0} \lim _{t \rightarrow \infty} E\left\{G\left(u^{e}(x, t, \omega)\right)\right\}=\lim _{t \rightarrow \infty} \lim _{\varepsilon \rightarrow 0} E\left\{G\left(u^{e}(x, t, \omega)\right)\right\} \\
= & \lim _{t \rightarrow \infty} E\left\{G\left(u_{0}(x, t)\right)\right\}=\lim _{t \rightarrow \infty} G\left(u_{0}(x, t)\right)=G\left(u^{*}\right) .
\end{array}
$$

Pincus [3] generalized the result of Schilder to a class of general Gaussian processes. The analysis given above can be duplicated for this case and will also extend to Gaussian processes with multidimensional parameters. 
Acknowledgement. I would like to thank Professor Henry McKean for his advice and guidance in the preparation of this manuscript.

Appendix. To complete the proof of Theorem 3 it is necessary to show that if

$$
J_{N} u=\int_{0}^{t} P(t-s) J_{N} f\left(J_{N} u\right) d s+\int_{0}^{t} P(t-s) J_{N} \alpha(s) d s+P(t) J_{N} u_{0}
$$

then the unique stationary distribution of $J_{N} u$ has the Radon-Nikodym derivative $\exp \left(2 F\left(J_{N} u\right)\right) / \int_{H} \exp \left(2 F\left(J_{N} u\right)\right) \delta p_{0}(u)$ with respect to $\mathfrak{p}_{0}(u)$ on $J_{N} H$.

Rewrite the equation in the form

$$
J_{N} u(t)=\int_{0}^{t}\left(J_{N} L J_{N} u(s)+J_{N} f\left(J_{N} u\right)\right) d s+\int_{0}^{t} J_{N} \alpha(s) d s+J_{N} u_{0} .
$$

Since $\int_{0}^{t} J_{N} \alpha(s) d s$ is an $N$-dimensional Wiener process, the equation is a finitedimensional Ito equation. The following theorem gives the stationary distribution explicitly for a wide class of Itô equations including the one above. Note that $J_{N} L J_{N} u+J_{N} f\left(J_{N} u\right)$ is Lipschitz continuous on $J_{N} H$ and also is the gradient of $L_{N} u \cdot J_{N} u+F\left(J_{N} u\right)$.

Theorem. Let $u(t) \in R^{N}$ satisfy $u(t)=\int_{0}^{t} f(u(s)) d s+W(t)+u(0)$ where $W(t)$ is an N-dimensional Wiener process. In addition let the following conditions be satisfied.

(i) $f$ is Lipschitz continuous.

(ii) $f(u)=\operatorname{grad} F(u)$ for some function $F$.

(iii) $\int_{R^{N}} e^{2 F(x)} d x<\infty$ where $d x$ is the differential of $N$-dimensional Lebesgue measure.

(iv) Prob $\{u(0) \in S\}=\int_{S} e^{2 F(x)} d x$ where $S$ is any measurable set in $R^{N}$.

Then for any $T>0$, Prob $\{u(T) \in S\}=\int_{S} e^{2 F(x)} d x$.

Proof of Theorem. The method of the proof is to find a sequence of processes $u_{R}(t)$ such that

$$
\begin{aligned}
\operatorname{Prob}\{u(T) \in S\} & =\lim _{R \rightarrow \infty} \operatorname{Prob}\left\{u_{R}(T) \in S\right\} \\
& =\int_{S} e^{2 F(x)} d x / \int_{R^{N}} e^{2 F(x)} d x .
\end{aligned}
$$

Let $u_{R}(t)$ with $\left|u_{R}\right|<R$ satisfy

$$
u_{R}(t)=\int_{0}^{t} f\left(u_{R}(s)\right) d s+W(t)+u_{R}(0)
$$

and

$$
\operatorname{Prob}\left\{u_{R}(0) \in S\right\}=\int_{S} e^{2 F(x)} d x / \int_{|x| \leq R} e^{2 F(x)} d x .
$$

Let $u_{R}(t)$ be reflected at the boundary $\left|u_{R}(t)\right|=R$.

Let $P_{R}(x, t)$ be defined by $\int_{S} P_{R}(x, t) d x=\operatorname{Prob}\left\{u_{R}(t) \in S\right\}$. $P_{R}(x, t)$ satisfies the forward Kolmogorov equation $\partial P_{R} / \partial t=\frac{1}{2} \operatorname{div} \operatorname{grad} P_{R}-\operatorname{div}\left(f(x) P_{R}\right)$. The 
side conditions are $P_{R}(x, 0)=e^{2 F(x)} / \int_{|x| \leq R} e^{2 F(x)} d x$, and at the boundary $|x|=R$ the normal component of $\frac{1}{2} \operatorname{grad} P_{R}(x, t)-f(x) P_{R}(x, t)$ is 0 . (The fact that the above is the correct boundary condition for the forward Kolmogorov equation with reflection at the boundary can be easily verified with the use of the divergence theorem.)

$$
P_{R}(x, t)=e^{2 F(x)} / \int_{|x| \leq R} e^{2 F(x)} d x
$$

is a solution to the Kolmogorov equation satisfying the side conditions. It can be shown that with the given side conditions the equation has a unique solution (Friedman [1]). Hence

$$
\operatorname{Prob}\left\{u_{R}(T) \in S\right\}=\int_{S} e^{2 F(x)} d x / \int_{|x| \leq R} e^{2 F(x)} d x .
$$

The next step in the proof is to show

$$
\lim _{R \rightarrow \infty} \operatorname{Prob}\left\{u_{R}(T) \in S\right\}=\operatorname{Prob}\{u(T) \in S\} .
$$

Note that the processes $u_{R}(t)$ and $u(t)$ are equivalent for all paths that do not reach the boundary. Hence

$$
\begin{gathered}
\lim _{R \rightarrow \infty}\left|\operatorname{Prob}\left\{u_{R}(T) \in S\right\}-\operatorname{Prob}\{u(T) \in S\}\right| \\
\leq \lim _{R \rightarrow \infty} \operatorname{Prob}\left\{\sup _{0 \leq i \leq T} u(t) \geq R\right\} .
\end{gathered}
$$

Therefore it is only necessary to show that $\lim _{R \rightarrow \infty} \operatorname{Prob}\left\{\sup _{0 \leq t \leq T} u(t) \geq R\right\}$ $=0$. But it is well known that this follows from the Lipschitz continuity of $f$.

Concluding the proof,

$$
\begin{aligned}
\operatorname{Prob}\{u(T) \in S\} & =\lim _{R \rightarrow \infty} \operatorname{Prob}\left\{u_{R}(T) \in S\right\} \\
& =\lim _{R \rightarrow \infty} \int_{S} e^{2 F(x)} d x / \int_{|x| \leq R} e^{2 F(x)} d x \\
& =\int_{S} e^{2 F(x)} d x / \int_{R^{N}} e^{2 F(x)} d x .
\end{aligned}
$$

\section{BIBLIOGRAPHY}

1. A. Friedman, Partial differential equations of parabolic type, Prentice-Hall, Englewood Cliffs, N.J., 1964. MR 31 \#6062.

2. M. Schilder, Some asymptotic formulas for Wiener integrals, Trans. Amer. Math. Soc. 125 (1966), 63-85. MR 34 \# 1770.

3. M. Pincus, Gaussian processes and Hammerstein integral equations, Trans. Amer. Math. Soc. 134 (1968), 193-214. MR 37 \#6994.

4. V. V. Baklan, Variational differential equations and Markov processes in Hilbert space, Dokl. Akad. Nauk SSSR 159 (1964), 707-710 = Soviet Math. Dokl. 5 (1964), 1553-1556. MR 30 \# 1547.

Department of Mathematics, Staten Island Communtty College, Staten Island, New YoRx 10301 